\title{
AOR
}

Selected Papers of \#AolR2020:

The $21^{\text {st }}$ Annual Conference of the

Association of Internet Researchers

Virtual Event / 27-31 October 2020

\section{THE 'SEARCH-FOR METHOD': EXAMINING INSTANCES OF SUPPORT IN FACEBOOK GROUPS FOR DONOR-CONCEIVED PEOPLE}

Giselle Newton

University of New South Wales, Australia

\section{Introduction}

This work in progress outlines the 'search-for method', a practical user-led tool for discussing instances of participation in Facebook groups. This paper reports on initial findings from applying the 'search-for method' to semi-structured interviews with administrators and members $(\mathrm{N}=30)$ of Facebook groups for donor-conceived people from across Australia. More specifically, I examine how the tool enabled participants to lead discussion around social aspects of support and discuss how their support needs changed over time. The interviews focused on Facebook groups for donor-conceived people, make up one component of a multi-method doctoral study exploring Australian donor-conceived adults' experiences, views and support needs.

\section{Background}

There are estimated to be 20,000-60,000 donor-conceived people in Australia. Limited research has been conducted internationally that foregrounds the perspectives of donor-conceived people and few empirical studies have explored the meanings ascribed to negotiating everyday life as a donor-conceived adult. Social media platforms have provided new opportunities for donor-conceived people to access information about gamete donors, the biological relatives of donors, and donor siblings. Within the field of donor conception, recent studies have explored how media platforms are being used to link donor siblings through donor sibling registries (Hertz \& Nelson, 2018) and Facebook groups for mothers of donor-conceived children (Andreassen, 2018). However, limited research has considered the role of peer support and communitybuilding between donor-conceived people in online contexts. With the advent of Facebook groups for donor-conceived people over a decade ago, this study considers how donor-conceived people create supportive relationships with peers through social media and how this form of social support may contribute to donor-conceived people's health and wellbeing.

\section{The 'search-for method'}

Suggested Citation (APA): Newton, G. (2020, October). The 'Search-For Method': Examining Instances of Support in Facebook Groups for Donor-Conceived People. Paper presented at AolR 2020: The 21 Annual Conference of the Association of Internet Researchers. Virtual Event: AolR. Retrieved from http://spir.aoir.org. 
The 'search-for method' was designed as a tool for qualitative interviews and involves inviting participants to search for their name in the search bar of a Facebook group, thereby retrieving data of all instances they have posted in the group. Participants are asked to scroll through retrieved posts and narrate what they recall about specific instances. The interviewer may probe the participant to discuss reactions, comments, suggestions or support from other members of the group and elaborate on how other members' responses made them feel. This method builds on approaches to user-led platform exploration in qualitative research such as the 'walkthrough method' (Light et al., 2018), a practical tool for systematically mapping applications and the 'media goalong method'(Jørgensen, 2016), a mobile ethnographic method in which participants verbally sketch an online environment as they navigate it. In the same vein, the 'scroll back method' by Robards \& Lincoln (2017) is an approach to examining participants' digital traces longitudinally via their Facebook Timelines. The 'search-for method', described in this paper, adapts the 'scroll back method' from a method to explore individual histories on Facebook Timelines to a method for examining how individual's experiences of Facebook groups are shaped by other members.

Applying the 'search-for' method to Facebook groups for donor-conceived people In this study, the semi-structured interviews began with general discussion about the Facebook groups for donor-conceived, exploring participants' impressions of and contributions to these groups. The 'search-for method' was adopted in the final stage of interviews as a tool for eliciting concrete examples of participation in the groups with the aim of investigating how support was enacted by peers. While donor-conceived members of Facebook groups have diverse needs, common concerns that arise in Facebook groups for donor-conceived people include queries about how to find donors or donor siblings, how to interpret DNA connections from commercial DNA testing platforms, how to navigate relationships with newfound genetic relatives and how to reform policy and legislation on donor conception. As the needs of donor-conceived people shift over time, the ways members use Facebook groups change accordingly. In the interviews, participants scrolled through their digital archives narrating personal stories from different periods throughout their membership in the group. The 'search-for method' enabled the participant and researcher, as co-analysts, to track and examine specific instances of participation and interaction in the group. More broadly, this approach illustrated how individual and collective donor-conceived identities emerged and evolved with and through online group platforms. In doing so, it provided a framework for understanding sociality between peers longitudinally.

\section{Empowering participants to lead interviews; protecting participant privacy}

User-led methods for platform exploration, such as the 'search-for method', empower participants to lead the discussion of their experiences and disrupt the hierarchy between researcher and participant in the interview setting. Like many closed Facebook groups, Facebook groups for donor-conceived people have rules governing the sharing of information posted. In the 'search-for method', the researcher is not directly accessing the group, rather the experiences participants share are up to their discretion, allowing them to retain ownership over their digital data. By scrolling through content on their own device, participants could decide how to frame their own stories of support and whether to disclose sensitive information or omit experiences they did not wish to discuss. This sense of control is particularly important in research on sensitive issues 
and has been found to be beneficial in research with young people (Albury \& Byron, 2016; Marston, 2019). However, at times scrolling can be confronting when upsetting or forgotten memories are recalled, or when digital traces do not align with the participants recollection (Robards \& Lincoln, 2017). Thus, researchers must take additional care in relation to the kinds of probing undertaken in interviews and have briefing and debriefing protocols in place with information on services participants can access if they experience discomfort or distress.

\section{Conclusion}

Facebook groups represent an important resource for donor-conceived peers to access information, seek advice and share their experiences with their peers. This paper has outlined the 'search-for method', a practical approach to analysing participation and interactions on Facebook groups longitudinally. In this paper the 'search-for method' was applied to the context of Facebook groups for donor-conceived people to determine how participants made sense of their digital archives and reflected on the social support available through Facebook groups. Through employing the 'search-for method', the participants and researcher were able to co-analyse concrete instances in which they reached out to the group and the set of responses and reactions to their post. This paper contributes to understandings of how digital affinities and peer intimacies develop in Facebook groups longitudinally.

\section{References}

Albury, K., \& Byron, P. (2016). Safe on My Phone? Same-Sex Attracted Young People's Negotiations of Intimacy, Visibility, and Risk on Digital Hook-Up Apps. Social Media + Society, 2(4), 205630511667288.

Andreassen, R. (2018). Mediated Kinship: Gender, Race and Sexuality in Donor Families. Routledge.

Hertz, R., \& Nelson, M. K. (2018). Random Families: Genetic Strangers, Sperm Donor Siblings, and the Creation of New Kin. Oxford University Press.

Jørgensen, K. M. (2016). The media go-along: Researching mobilities with media at hand. MedieKultur: Journal of Media and Communication Research, 32(60), 18.

Light, B., Burgess, J., \& Duguay, S. (2018). The walkthrough method: An approach to the study of apps. New Media \& Society, 20(3), 881-900.

Marston, K. (2019). Researching LGBT+ Youth Intimacies and Social Media: The Strengths and Limitations of Participant-Led Visual Methods. Qualitative Inquiry, 11.

Robards, B., \& Lincoln, S. (2017). Uncovering longitudinal life narratives: Scrolling back on Facebook. Qualitative Research, 17(6), 715-730. 